the CAPTURE study mitigates the introduction of this new technology in clinical practice. Well designed clinical trials obtain their credibility from the definition of a priori hypotheses that helps researchers to avoid drawing wrong conclusions, and negative results are as useful as positive results in guiding medical treatments.

However, even when the primary outcome of a clinical trial fails, new research opportunities open up. ${ }^{8}$ Hopefully, a careful analysis of the CAPTURE data will lead to future research in the field. Additional laboratory studies are probably required to i) gain further insight into the damage that UV-C irradiation causes to platelets apart from pathogen inactivation and to ii) develop strategies to improve the quality of Theraflex treated products.

Concerns regarding the possible transfusion transmission of SARS-CoV-2 at the beginning of the ongoing pandemic have revamped the interest in approaches capable of protecting the blood supply from known and newly emerging threats. ${ }^{9}$ As Brixen and colleagues remind us, safe and effective pathogen reduction methods are still an unmet need.

\section{Disclosures}

$D P$ sits on advisory boards, has received travel or research grants, as well as speaking and teaching fees from Macopharma,
Ortho Clinical Diagnostics, Grifols, Terumo, Immucor, Diamed, Diatech Pharmacogenetics.

\section{References}

1. Heddle NM, Cardoso M, van der Meer PF. Revisiting study design and methodology for pathogen reduced platelet transfusions: a round table discussion. Transfusion. 2020;60(7):1604-1611.

2. Rebulla P, Garban F, van der Meer PF, Heddle NM, McCullough J. A crosswalk tabular review on methods and outcomes from randomized clinical trials using pathogen reduced platelets. Transfusion. 2020;60(6):1267-1277.

3. Rebulla $\mathrm{P}$. The long and winding road to pathogen reduction of platelets, red blood cells and whole blood. Br J Haematol. 2019;186(5):655-667

4. Estcourt LJ, Malouf R, Hopewell S, et al. Pathogen-reduced platelets for the prevention of bleeding. Cochrane Database Syst Rev. 2017;7(7):CD009072

5. Brixner V, Bug G, Pohler P, et al. Efficacy of UVC-treated, pathogenreduced platelets versus untreated platelets: a randomized controlled non-inferiority trial. Haematologica. 2021;106(4):1086-1096.

6. Rebulla P, Vaglio S, Beccaria F, et al. Clinical effectiveness of platelets in additive solution treated with two commercial pathogen-reduction technologies. Transfusion. 2017;57(5):1171-1183.

7. Mauri L, D'Agostino RB Sr. Challenges in the design and interpretation of noninferiority trials. N Engl J Med. 2017;377(14):1357-1367.

8. Pocock SI, Stone GW. The primary outcome fails - what next? N Engl J Med. 2016;375(9):861-870.

9. Stanworth SJ, New HV, Apelseth TO, et al. Effects of the COVID-19 pandemic on supply and use of blood for transfusion. Lancet Haematol. 2020;7(10):e756-e764.

\title{
Understanding how retinoic acid derivatives induce differentiation in non-M3 acute myelogeneous leukemia
}

\author{
Martin Carroll \\ Division of Hematology and Oncology, University of Pennsylvania, Philadelphia, PA, USA \\ E-mail: MARTIN CARROLL - carroll2@pennmedicine.upenn.edu
}

doi:10.3324/haematol.2020.275412

$\mathrm{O}$ ver 30 years ago, Huang and colleagues published the startling result that all trans-retinoic acid (ATRA) could induce clinical remissions without myelosuppression in patients with acute promyelocytic leukemia (APML). ${ }^{1}$ Analysis in this report and subsequent analysis demonstrated that responses are due to induced differentiation of the leukemic clone and not the induction of cell death in the malignant cells. This work introduced the concept of differentiation therapy to the world of leukemia therapeutics. Other recently developed therapeutics for acute myeloid leukemia (AML) including FLT3 and IDH inhibitors in some patients with the targeted mutations are now known to induce differentiation. ${ }^{2,3}$ However, there remain two outstanding questions in this field that stem from those original remarkable observations. First, what is the role of retinoic acid or its derivatives in controlling normal myeloid maturation? Second, how can this information be used to develop retinoic acid based therapeutics for non-M3 AML? di Martino and colleagues provide exciting new insights into these questions in this issue of Haematologica. ${ }^{4}$

To understand the complexity of these questions, it is valuable to first briefly introduce how retinoids and their derivatives function. Conceptually, retinoic acid (RA) functions through one of the retinoic acid receptors (RAR) which are members of the nuclear hormone receptor family. To simplify, binding of RA to the RAR induces binding to DNA. Commonly, this binding leads to recruitment of factors that promote gene transcription (such as histone acetyl transferases) and displacement of inhibitors of transcription such as nuclear receptor corepressor (NCOR1). There are many levels of complexity in these gene regulatory events. ${ }^{5}$ Importantly, there are actually three isoforms of RAR. RAR can function as homodimers, heterodimers with themselves or heterodimers with other members of the nuclear hormone receptor superfamily including retinoic X receptors (RXR), Vitamin $\mathrm{D}$ receptors (VDR) and peroxisome proliferator activated receptor (PPAR). Thus, there are many combinatorial possibilities for gene targets and multiple levels of redundancy that have made defining the specific role of the nuclear hormone receptor superfamily in myeloid maturation and leukemia therapy challenging.

To address these questions, Di Martino and colleagues first use a murine model of AML induced using the KMT2A fusion protein, KMT2A-AF9. The leukemic cells were transduced with reporter constructs that are quite specific for activation by isoforms of RAR or RXR, trans- 
planted into secondary recipients and reporter activation was used to determine if the leukemias have endogeneous expression of RXR or RAR ligands. These results show that the AML cells studied have spontaneous activation of RXRA which can be further stimulated with the RXR agonist, bexarotene. In contrast, there is minimal endogeneous activation of RARA. This is consistent with the group's previous report studying stress myelopoiesis. ${ }^{6}$ To further study the role of RXRA and RXRB in this AML model the authors planned to induce leukemia in cells engineered to have a deletable form of the receptors. In a case of remarkably informative serendipity, the authors demonstrate robust selection in the leukemias for spontaneous deletion of RXRA and RXRB. Elegantly designed experiments validate that the RXR complex is a tumor suppressor in this model of AML. This better defines the biology of RXR in AML but targeting of RXR, as further discussed below, has not been adequate to induce a robust response in AML.

However, the authors continue on to studies that inform a pharmacologic approach to treatment of AML. Bexarotene is a small molecular agonist of RXR that Tsai and colleagues previously demonstrated to have modest activity in non-M3 AML.? diMartino and colleagues confirm that RXR activity stimulated by bexarotene has in vitro activity decreasing leukemic cell growth and that this is synergistically stimulated by simultaneous activation of RAR using ATRA or the more specific RARA ligand, BMS753. ${ }^{\circ}$ They then use the RXRA deleted cells generated in vivo, re-introduce wild type or mutant RXRA and define the domains of RXRA necessary for suppressing leukemogenesis. This experiment is the most molecular robust definition of the requirement for RXR activation to reduce leukemogenesis to date and convincingly demonstrates that bexarotene effects are not off target effects. An interesting Figure 4 both validates this conclusion and demonstrates that the effects of retinoid/rexinoid combinations on leukemic cells depend strongly on the cell culture system. To further define the molecular effects of RAR and RXR on leukemic cells, the authors use reporter assays to show that RARA can have a strong interaction with the nuclear co-repressor, silencing mediator of retinoic acid and thyroid hormone receptor (SMRT). Overall, these data suggest that the therapeutic target complex in AML cells is a RARA/RXRA heterodimer which can act as a nuclear repressor. The ATRA-dependent release of corepressor from the RARA:RXRA heterodimer may account for the synergy described. Simultaneous stimulation of both RARA with ATRA and RXR with bexarotene works best to induce this repressive activity and induce leukemic cell death or differentiation.

Importantly, for translational use, diMartino and colleagues go on to validate efficacy of the drug combination in multiple murine and human models of AML including primary human AML cells studied in culture. The clinical development of this drug combination is challenging. The authors have used, among other compounds, a bexarotene derivative, CW103-4 which has both RARA and RXRA agonist activity and, in theory, should be a potent anti-leukemic drug. However, CW103-4 is reported to be toxic in humans due to the induction of hypertriglyderidemia. ${ }^{9}$ Of note, careful experiments shown here confirm that ATRA and bexarotene combinations are quite toxic in mice making it difficult to use the mouse model to test novel rexinoid drug combinations for AML therapy. However, the results here do suggest, for the first time, a way forward in this difficult area. Based on these studies, we can feel confident that the target complex in AML is a RARA: RXRA heterodimer. The therapeutic effects of the ATRA:bexarotene combination is likely achieved through changes in gene expression. Use of the carefully developed murine models shown here should allow for combinations of chromatin immunoprecipitation sequencing (ChiP-seq) and RNA seq studies that may define targets of the drug combination which can be pharmacologically targeted leading to non-toxic differentiation therapy for non-M3 AML. Alternatively, very careful drug binding studies may allow for dissociating the triglyceride regulating effects of retinoids and rexinoids from the myeloid effects. Either one of these approaches may bring us closer to the longsought goal of differentiation therapy for non-M3 AML.

\section{Disclosures \\ No conflicts of interest to disclose.}

\section{References}

1. Huang ME, Ye YC, Chen SR, et al. Use of all-trans retinoic acid in the treatment of acute promyelocytic leukemia. Blood. 1988;72(2):567 572 .

2. McMahon CM, Canaani J, Rea B, et al. Gilteritinib induces differentiation in relapsed and refractory FLT3-mutated acute myeloid leukemia. Blood Adv. 2019;3(10):1581-1585.

3. Fathi AT, DiNardo C, Kline I, et al. Differentiation syndrome associated with enasidenib, a selective inhibitor of mutant isocitrate dehydrogenase 2: analysis of a phase 1/2 study. JAMA Oncol. 2018; 4(8):1106-1110

4. di Martino O, Niu H, Hadwiger G, et al. Endogenous and combination retinoids are active in myelomonocytic leukemias. Haematologica. 2021;106(4):1008-1021.

5. le Maire A, Alvarez S, Shankaranarayanan P, de Lera AR, Bourguet W, Gronemeyer H. Retinoid receptors and therapeutic applications of RAR/RXR modulators. Curr Top Med Chem. 2012;12(6):505-527.

6. Niu H, Fujiwara $\mathrm{H}$, di Martino $\mathrm{O}$, et al. Endogenous retinoid X receptor ligands in mouse hematopoietic cells. Sci Signal. 2017;10(503):eaan1011.

7. Tsai DE, Luger SM, Andreadis C, et al. A phase I study of bexarotene, a retinoic $\mathrm{X}$ receptor agonist, in non- $\mathrm{M} 3$ acute myeloid leukemia. Clin Cancer Res. 2008;14(17):5619-5625.

8. Sanchez PV, Glantz ST, Scotland S, Kasner MT, Carroll M. Induced differentiation of acute myeloid leukemia cells by activation of retinoid X and liver X receptors. Leukemia. 2014;28(4):749-760.

9. Marshall PA, Jurutka PW, Wagner CE, et al. Analysis of differential secondary effects of novel rexinoids: select rexinoid $X$ receptor ligands demonstrate differentiated side effect profiles. Pharmacol Res Perspect. 2015;3(2):e00122. 